\title{
Compositional Construction of Bounded Error Over-Approximations of Acyclic Interconnected Continuous Dynamical Systems
}

\author{
Ratan Lal \\ Kansas State University \\ ratan@ksu.edu
}

\author{
Pavithra Prabhakar \\ Kansas State University \\ pprabhakar@ksu.edu
}

\begin{abstract}
We consider the problem of bounded time safety verification of interconnections of input-output continuous dynamical systems. We present a compositional framework for computing bounded error approximations of the complete system from those of the components. The main crux of our approach consists of capturing the input-output signal behaviors of a component using an abstraction predicate that represents the input-output sample behaviors corresponding to the signal behaviors. We define a semantics for the abstraction predicate that captures an over-approximation of the input-output signal behaviors of a component. Next, we define how to compose abstraction predicates of components to obtain an abstraction predicate for the composed system. We instantiate our compositional abstraction construction framework for linear dynamical systems by providing concrete methods for constructing the input-output abstraction predicates for the individual systems.
\end{abstract}

\section{CCS CONCEPTS}

- General and reference $\rightarrow$ Verification; • Theory of computation $\rightarrow$ Logic and verification; Abstraction.

\section{KEYWORDS}

Continuous Systems, Approximation, Composition, Safety

\section{ACM Reference Format:}

Ratan Lal and Pavithra Prabhakar. 2019. Compositional Construction of Bounded Error Over-Approximations of Acyclic Interconnected Continuous Dynamical Systems. In 17th ACM-IEEE International Conference on Formal Methods and Models for System Design (MEMOCODE '19), October 9-11, 2019, La folla, CA, USA. ACM, New York, NY, USA, 5 pages. https://doi.org/10. $1145 / 3359986.3361210$

\section{INTRODUCTION}

Safety is an important property required of software that controls physical systems as in autonomous vehicles, process control systems and medical devices. Hybrid systems $[1,18]$ are a popular formalism to model the interactions between discrete (software) and continuous (physical) systems. Safety verification consists of

Permission to make digital or hard copies of all or part of this work for personal or classroom use is granted without fee provided that copies are not made or distributed for profit or commercial advantage and that copies bear this notice and the full citation on the first page. Copyrights for components of this work owned by others than ACM must be honored. Abstracting with credit is permitted. To copy otherwise, or republish, to post on servers or to redistribute to lists, requires prior specific permission and/or a fee. Request permissions from permissions@acm.org.

MEMOCODE '19, October 9-11, 2019, La folla, CA, USA

(ㄷ) 2019 Association for Computing Machinery.

ACM ISBN 978-1-4503-6997-8/19/10 . \$ \$15.00

https://doi.org/10.1145/3359986.3361210 checking if all the executions of the system remain within a safety zone. This is typically accomplished by computing the reach set of the the system that consists of all states reached by the system executions; and then checking that the reach set does not intersect with the unsafe zone. The challenging part of safety verification of hybrid systems lies in computing the reachable set of the continuous dynamics capturing the physical system behaviors. In this paper, we focus on safety verification of continuous dynamical systems.

The reach set computation problem consists of computing a representation of the reach set such that the problem of checking the emptiness of its intersection with the unsafe zone is decidable. In general, computing the exact reach set is challenging, and is feasible only for certain simple classes of dynamics such as constant rate and rectangular/polyhedral inclusion dynamics [17]. For more complex dynamical systems such as linear dynamical systems, the solutions evolve exponentially with time and hence, require exponential functions to represent the reachable set; checking the safety requires solving constraints involving exponential functions for which there are no known decision procedures [32]. Hence, the research over the past decade has focused on computing overapproximations of the reach set each of which provides a certain trade-off between computational complexity and precision of overapproximation. Broadly, the techniques can be categorized into two classes, namely, flowpipe computation techniques and abstraction techniques. Flowpipe computation [7, 10, 13, 14, 19, 25] consists of computing the solutions of the dynamical system at certain sample time points and using these values to compute an envelope for the reach set. Iteratively precise over-approximation can be obtained by reducing the sampling interval. On the other hand, abstraction techniques construct a simpler abstract system such that the reach set in the abstract system corresponds to an over-approximation of the actual reach set. These techniques, in general, do not provide bounds on the precision of over-approximation, however, they are often accompanied by refinement loops that aim to construct iteratively qualitatively better abstractions [2-4, 11, 12, 26, 27].

The main focus of this paper is to develop a compositional framework for the construction of over-approximations of the system behaviors that have bounded error with respect to the original behaviors. The main motivation for this work is the compositional design of systems, wherein we build systems by composing certain components. Assuming that each of the components comes with a correctness proof (in our case an input-output behavior guarantee), we need to be able to exploit these to provide a correctness proof of the composed system, instead of finding proof from scratch. In addition, if a component changes, then we could reuse the proofs 
for the rest of the components and recompute only the proof of the component that changed, and finally, compose them all. On the flip side, the compositional construction might have its one time overhead which might make it slightly more computationally expensive. In this paper, we focus on over-approximating the input-output behaviors rather than the reach set, since, even if the composed system is a closed system (system with no inputs), the individual components might have inputs and outputs through which they interact with other components. It is straightforward to obtain an over-approximation of the reach set of a closed system from an over-approximation of the input-output behavior.

There are several challenges to the design of a compositional over-approximation computation framework. Consider, for instance, a system that consists of two components $\dot{x}(t)=f(x(t), y(t))$ and $\dot{y}(t)=g(y(t), z(t))$ where the dot represent the derivative with respect to time. Note that the behaviors of this system are continuous trajectories $x, y, z:[0, T] \rightarrow \mathbb{R}$. The first challenge is an effective representation of the input-output continuous behaviors. We choose to capture the behaviors of a component $\mathcal{S}$ using a predicate $\phi$ whose free variables capture the values of the input and output trajectories at certain sample times. However, $\phi$ only captures discrete behaviors rather than continuous behaviors. Hence, we define a semantics for $\phi$ which captures a set of continuous behaviors. More precisely, given positive real numbers $\theta, \epsilon$ and $r$, we define $\llbracket \phi \rrbracket^{\theta, \epsilon, r}$ to be a set of input-output trajectories $(u, y)$ such that the rate of change of input trajectory $u$ is bounded by $\theta$, the sampled values of $u$ and that of some signal $y^{\prime}$ which is within $\epsilon$ of $y$ satisfy the formula $\phi$ when sampled at intervals of size $r$. The motivation for the semantics is as follows. Every input-output sequence $(\bar{u}, \bar{y})$ that satisfies $\phi$ is intended to capture a set of trajectories. There are uncountably many trajectories that have $\bar{u}$ as a sampled sequence; however, not all of the corresponding output trajectories will have $\bar{y}$ as the sampled trace. However, if we restrict the input trajectories to have abounded deviation, specified using rate $\theta$, from a piecewise linear trajectory corresponding to $\bar{u}$, then the output trajectories will also be bounded by some $\epsilon$ with respect to the output trajectory of the piecewise continuous input signal. There are many choices to capture input-output deviations, this is one of the choices for which the compositional construction works.

Our main results are the following. First we define a predicate $\phi_{\mathcal{S}}^{r}$ (Definition 8) that captures a set of input-output sequences with sample interval $r$ depending on $\mathcal{S}$ such that $\llbracket \phi \rrbracket^{\theta, \epsilon, r}$ is a bounded error over-approximation of $\mathcal{S}$. This is formally stated in Theorem 1 using certain functions that capture the input-output deviations of the system $\mathcal{S}$. Next, we provide a definition of composition of predicates $\phi_{\mathcal{S}_{1}}^{r}$ and $\phi_{\mathcal{S}_{2}}^{r}$ (Definition 12) such that the composed predicate is a bounded error over-approximation of the input-output behaviors of the composition of $\mathcal{S}_{1}$ and $\mathcal{S}_{2}$ (Theorem 2). Finally, we instantiate the framework for the class of linear dynamical systems, that is, we provide a specific construction for $\phi_{\mathcal{S}}^{r}$ which satisfies Definition 8. Also, we provide an algorithm that takes as input an error bound $\gamma$ and computes a $\gamma$ bounded input-output overapproximation of a composed system by inductively computing the same for its components. We have analyzed two case studies based on these results to demonstrate the feasibility of the approach.
Related work. Compositional modeling of hybrid systems has been explored in the realm of hybrid input-output automata [22]. Compositional analysis based on theorem proving has been studied in [24] for hybrid systems. Compositional analysis based on assume guarantee reasoning $[9,21]$ as well as methods for learning assumptions $[5,8,23]$ have been explored. In addition, there is some recent work on assume guarantee reasoning for linear hybrid systems based on hybridization [6]. Also, simulation based compositional analysis $[15,16]$ of interconnected systems and compositional abstraction [28-31] of hybrid systems have been studied. However, in general, these methods do not construct bounded error approximations, which is the objective of the work in this paper. Although compositional bounded error approximation [20] has been studied for hybrid systems, it is not applicable for interconnected dynamical systems.

\section{MOTIVATION}

Complex systems consist of multiple components that interact with each other. Each component can be interpreted as a system that transforms certain input signals into output signals; we refer to these as input-output systems (IOSs). The components are interconnected through input/output variables as shown in Figure 1.

There are two input-output systems $\mathcal{S}_{1}$ and $\mathcal{S}_{2} ; \mathcal{S}_{1}$ has 3 input variables $u_{1}, u_{2}$ and $u_{3}$ and 3 output variables $y_{1}, y_{2}$ and $y_{3}$, and $\mathcal{S}_{2}$ has 4 input variables $u_{1}, y_{2}, y_{3}$ and $u_{4}$ and 2 output variables $y_{4}$ and $y_{5}$. The interconnection is implicitly specified using common variable names. Hence, in this composition, the two outputs $y_{2}$ and $y_{3}$ of $\mathcal{S}_{1}$ are connected to inputs $y_{2}$ and $y_{3}$ of $\mathcal{S}_{2}$, respectively. The composed system has as inputs $u_{1}, u_{2}, u_{3}$ and $u_{4}$, namely, the inputs of the first system, and the inputs of the second system which are not connected to any outputs of the first system. The outputs of the composed system are $y_{4}, y_{5}$ and $y_{1}$, which correspond to the outputs of the second system and the outputs of the first system that are not connected to the inputs of the second system.

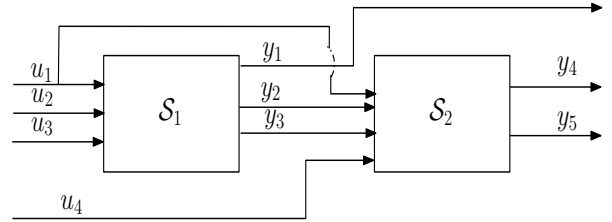

Figure 1: Composition of input-output systems

The composed system has two special subcases, namely, serial and parallel composition wherein serial, the set of output variables of the first system is the same as the input variables of the second system in serial, and in parallel, the input variables for the two systems are the same, however, the output variables of the first system and the input variables of the second system are disjoint. A widely prevalent class of composed systems consist of plant and control that are connected through a feedback loop. However, here we focus on systems that are built compositionally from smaller components, and assume that the interconnections do not have loops.

\section{PRELIMINARIES}

Numbers and Functions. Let $\mathbb{R}$ be the set of real numbers and $[n]$ be the set $\{1,2, \ldots, n\}$. We use $\operatorname{dom}(x)$ for the domain of the 
variable $x$. Given function $h:[0, T] \rightarrow A, r \geq 0$ such that $T=r n$, we define a set of points of the function $h$ at $i r, i=0,1, \ldots, n$ as a tuple $[h]_{r}=\left(h(0), h(r), h(2 r), \ldots, h\left(\frac{T}{r}\right)\right)$. Given tuple $g=$ $\left(g_{0}, g_{1}, \ldots, g_{n}\right)$, where $g_{i} \in A, T \geq 0$ such that $T=r n$, we define a piece-wise constant function $\langle g\rangle_{r}:[0, T] \rightarrow A$ such that $\langle g\rangle_{r}(t)=$ $g_{i}$, ir $\leq t<(i+1) r$, for $i=0,1, \ldots, n-1,\langle g\rangle_{r}(T)=g_{n}$. For $x \in \mathbb{R}^{n}$, we use $\|x\|=\max \left\{\left|x_{1}\right|, \ldots,\left|x_{n}\right|\right\}$.

Concatenation. Given two tuples $x=\left(x_{1}, x_{2}, \ldots, x_{n}\right)$ and $y=$ $\left(y_{1}, y_{2}, \ldots, y_{m}\right)$, we use $x \frown y$ to denote the concatenation of $x$ and $y$ which is defined as follows: $x \frown y=\left(x_{1}, x_{2}, \ldots, x_{n}, y_{1}, y_{2}, \ldots, y_{m}\right)$. It can be extended for sets.

Projection. Given tuple $x=\left(x_{1}, x_{2}, \ldots, x_{n}\right)$, and an index set $I \subseteq[n]$, we use $x L_{I}$ to denote the projection of the tuple $x$ with respect to the index set $I$, for example, $x L_{I}=\left(x_{i_{1}}, x_{i_{2}}, \ldots, x_{i_{p}}\right)$, where $1 \leq i_{1}<i_{2}<\ldots<i_{p} \leq n$ and $I=\left\{i_{1}, \cdots, i_{p}\right\}$. It can be extended for sets.

\section{SYSTEM DESCRIPTION}

In this section, we provide the formal definition of the interconnected systems that we study. In this paper, we consider compositions that do not lead to feedback loops.

\subsection{Input-output systems}

We formally define an input-output system that models a continuous time system using a set of pairs of input-output trajectories.

Definition 1. Given a set $\mathcal{A}$, a trajectory over $\mathcal{A}$ is a function $\tau:[0, T] \rightarrow \mathcal{A}$ for some $T \geq 0$. We represent $\operatorname{Traj}(A)$ to denote the set of trajectories over the set $\mathcal{A}$.

Definition 2. Given a set $\mathcal{A}$ and $r \geq 0$, a piece-wise constant trajectory with sample interval $r$ is a function $\tau:[0, T] \rightarrow \mathcal{A}$, for some $T \geq 0$ such that $T=r n$ for some natural number $n$ and for every $i \in[n]$, there exists $c_{i}$ such that $\tau(t)=c_{i}$ for all $(i-1) r \leq t<i r$. Let $\operatorname{Traj}_{r}(\mathcal{A})$ represent the set of all piece-wise constant trajectories over the set $\mathcal{A}$ with sample interval $r$.

Definition 3. An input-output system (IOS) is a tuple $\mathcal{S}=(I, O$, $\mathcal{U}, \mathcal{Y}, \mathcal{T})$, where

(a) $I=\left(u_{1}, u_{2}, \ldots, u_{m}\right)$ is a tuple of input variables;

(b) $O=\left(y_{1}, y_{2}, \ldots, y_{n}\right)$ is a tuple of output variables;

(c) $\mathcal{U}=U_{1} \times U_{2} \times \ldots \times U_{m}$ is the input domain;

(d) $\mathcal{Y}=Y_{1} \times Y_{2} \times \ldots \times Y_{n}$ is the output domain;

(e) $\mathcal{T} \subseteq \operatorname{Traj}(\mathcal{U}) \times \operatorname{Traj}(\mathcal{Y})$ is a set of input-output trajectories such that for all $(u, y) \in \mathcal{T}, \operatorname{dom}(u)=\operatorname{dom}(y)$.

In the above definitions $U_{i}$ corresponds to the input domain of the variable $u_{i}$, and $Y_{i}$ to that of $y_{i}$. We will often refer to an input-output system as just system.

Next, we illustrate how to model a linear system as an inputoutput system. Consider a linear system,

$$
\dot{x}(t)=A x(t)+B u(t), \quad y=C x(t)
$$

where $x(t) \in X \subseteq \mathbb{R}^{n}, u(t) \in U \subseteq \mathbb{R}^{m}$, and $y(t) \in Y \subseteq \mathbb{R}^{p}$, where $X, U$ and $Y$ are compact sets; $X_{0} \subseteq X$ is an initial convex compact polyhedral set. Here $A \in \mathbb{R}^{n \times n}, B \in \mathbb{R}^{n \times m}$ and $C \in \mathbb{R}^{p \times n}$ are state, input, and output matrices, respectively. The input-output system corresponding to the System (1) up to a time bound $T$ is given by $\mathcal{S}=(I, O, \mathcal{U}, \mathcal{Y}, \mathcal{T})$, where (a) $I=\left(u_{1}, u_{2}, \ldots, u_{m}\right) ; O=\left(y_{1}, y_{2}, \ldots, y_{p}\right)$;

(b) $\mathcal{U}=U ; \quad y=Y$;

(c) $\mathcal{T}=\left\{(u, y) \in \operatorname{Traj}(\mathcal{U}) \times \operatorname{Traj}(\mathcal{Y}) \mid \operatorname{dom}(u)=\operatorname{dom}(y)\right.$ and $\exists x_{0} \in$ $X_{0}, y(t)=C x(t)$, where $\left.x(t)=e^{A t} x_{0}+\int_{0}^{t} e^{A\left(t-t^{\prime}\right)} B u\left(t^{\prime}\right) d t^{\prime}\right\}$.

Assumption 1. We assume that for every input trajectory $u \in$ $\operatorname{Traj}(\mathcal{U})$, there is an output trajectory $y \in \operatorname{Traj}(\mathcal{Y})$ such that $(u, y) \in$ $\mathcal{T}$. It is not restrictive because most continuous dynamical systems have this property.

\subsection{Composition of input-output systems}

In this section, we define the composition of input-output systems. We use $\mathcal{S}_{1} \triangleright \mathcal{S}_{2}$ to denote the composition of IOSs $\mathcal{S}_{1}$ and $\mathcal{S}_{2}$. First, we define the conditions under which two input-output systems can be composed; this will eliminate the possibility of feedback loops in the system.

Definition 4. An input-output system $\mathcal{S}_{1}=\left(I_{1}, O_{1}, \mathcal{U}_{1}, \mathcal{Y}_{1}, \mathcal{T}_{1}\right)$ can be composed with another input-output system $\mathcal{S}_{2}=\left(I_{2}, \mathrm{O}_{2}, \mathcal{U}_{2}\right.$, $\left.\mathcal{Y}_{2}, \mathcal{T}_{2}\right)$ if following conditions hold:

(a) $\llbracket I_{1} \rrbracket \cap \llbracket O_{2} \rrbracket=\emptyset ;(b) \llbracket O_{1} \rrbracket \cap \llbracket O_{2} \rrbracket=\emptyset$.

Next, we construct the composition of two input-output systems.

Definition 5. Given two input-output systems $\mathcal{S}_{i}=\left(I_{i}, O_{i}\right.$, $\left.\mathcal{U}_{i}, \mathcal{Y}_{i}, \mathcal{T}_{i}\right), i=1,2$, which can be composed, the composed system is $\mathcal{S}_{1} \triangleright \mathcal{S}_{2}=(I, O, \mathcal{U}, \mathcal{Y}, \mathcal{T})$, where

(a) $I=I_{1} \frown I_{2} l_{\operatorname{diff}\left(I_{2}, O_{1}\right)} ; O=O_{1} l_{\operatorname{diff}\left(O_{1}, I_{2}\right)} \frown O_{2}$;

(b) $\mathcal{U}=\left.\mathcal{U}_{1} \frown \mathcal{U}_{2}\right|_{\text {diff }\left(I_{2}, O_{1}\right)} ; \boldsymbol{Y}=\left.\mathcal{Y}_{1}\right|_{\text {diff }\left(O_{1}, I_{2}\right)} \frown \mathcal{Y}_{2}$;

(c) $\mathcal{T}=\left\{(u, y) \mid \exists\left(u_{1}, y_{1}\right) \in \mathcal{T}_{1},\left(u_{2}, y_{2}\right) \in \mathcal{T}_{2}, u=u_{1} \frown u_{2} l_{\text {diff }\left(I_{2}, O_{1}\right)}\right.$, $\left.y=y_{1} l_{\text {diff }}\left(O_{1}, I_{2}\right) \frown y_{2}\right\}$.

REMARK 1. The composition $\mathcal{S}_{1} \triangleright \mathcal{S}_{2}$ of two input-output systems $\mathcal{S}_{1}$ and $\mathcal{S}_{2}$ is a serial composition if $I_{2}=O_{1}$ and $\mathcal{U}_{2}=\mathcal{Y}_{1}$, and it is a parallel composition if $I_{1}=I_{2}, \mathcal{U}_{1}=\mathcal{U}_{2}$, 【I $\rrbracket \cap \llbracket O_{1} \rrbracket=\emptyset$ and $\llbracket I_{1} \rrbracket \cap \llbracket O_{2} \rrbracket=\emptyset$.

\subsection{Bounded Error Approximation}

Our objective is to compute bounded error over-approximations for input-output behaviors of systems. This computation captures more input-output behaviors than the input-output behaviors of the systems, which are defined by the notion of $\gamma$ perturbation of the behaviors. We will restrict ourselves to input trajectories whose rate is bounded by some assigned value $\theta$.

Definition 6. Given system $\mathcal{S}, \theta \geq 0$, and $\gamma \geq 0$, we define $\llbracket \mathcal{S} \rrbracket^{(\theta, \gamma)}$ to be the set of all input-output signals $(u, y)$ such that $\|\dot{u}\| \leq \theta$ and $y$ is within $\gamma$ of $y^{\prime}$, where $\left(u, y^{\prime}\right) \in \mathcal{T}$. In other words,

$$
\llbracket \mathcal{S} \rrbracket^{(\theta, \gamma)}=\left\{(u, y) \mid\|\dot{u}\| \leq \theta, \exists\left(u, y^{\prime}\right) \in \mathcal{T},\left\|y-y^{\prime}\right\| \leq \gamma\right\} .
$$

When $\gamma=0$, we use $\llbracket \mathcal{S} \rrbracket^{\theta}$ instead of $\llbracket \mathcal{S} \rrbracket^{(\theta, 0)}$.

Hence, a $\gamma$-bounded over-approximation is any set of inputoutput behaviors that contains $\llbracket \mathcal{S} \rrbracket^{\theta}$ but is contained in $\llbracket \mathcal{S} \rrbracket^{(\theta, \gamma)}$.

\section{COMPOSITIONAL FRAMEWORK FOR ABSTRACTION CONSTRUCTION}

In this section, we present our compositional framework to construct an abstraction of system executions over a bounded time by 
using the abstractions of the components. The crux of our construction relies on an abstraction predicate that captures the sampled input-output behaviors of a system. In Section 5.1, we formalize the notion of an abstraction predicate, and show how it can be used to capture bounded error over-approximations of input-output behaviors of component systems. Next, in Section 5.2, we present how to compose abstraction predicates of components to obtain abstraction predicates for the composed system.

\subsection{Abstraction of Components}

The broad goal of this section is to define a bounded error overapproximation of input-output behaviors of a system $\mathcal{S}$, where the rate of change of input is bounded by some value $\theta$. In other words, given an error bound $\gamma$, we intend to compute a set of input-output behaviors $\mathcal{S}^{\prime}$ that contains $\llbracket \mathcal{S} \rrbracket^{\theta}$, but is contained in $\llbracket \mathcal{S} \rrbracket^{(\theta, \gamma)}$. Our broad approach towards defining $\mathcal{S}^{\prime}$ is to define a predicate $\phi_{\mathcal{S}}^{r}$ that captures the input-output sample behaviors of $\mathcal{S}$ and define a semantics $\llbracket \phi_{\mathcal{S}}^{r} \rrbracket^{(\theta, \epsilon, r)}$ that provides the required $\mathcal{S}^{\prime}$ In particular, $\llbracket \phi_{\mathcal{S}}^{r} \rrbracket^{(\theta, \epsilon, r)}$ is a $\gamma$ bounded over-approximation of $\mathcal{S}$ only for certain values of $\epsilon$ and $r$ for a fixed value of $\theta$ and $\gamma$. In order to obtain the values of $\epsilon$ and $r$, we use certain properties of the dynamics $\mathcal{S}$ which are captured using auxiliary functions $C_{\mathcal{S}}$ and $\mathcal{B}_{\mathcal{S}}$. Hence, we formally define these notions below.

We use formula $\varphi(\overline{\mathbf{u}}, \overline{\mathbf{y}})$ to denote a first-order logic predicate with free variables $\overline{\mathbf{u}}$ and $\overline{\mathbf{y}}$, each of which represents a sequence of free variables. We will assume that the sequences are of the same length. Our results in this section are not affected by the specific vocabulary of the predicates, however, it will become important when we want a decision procedure for safety verification. We assume that there is a standard semantics for the predicate $\varphi(\overline{\mathbf{u}}, \overline{\mathbf{y}})$ which consists of pair of sequences of real values for the variables $\overline{\mathbf{u}}$ and $\overline{\mathbf{y}}$ for which the predicate is evaluated as true. We use $\llbracket \varphi \rrbracket_{p w}$ to denote the standard semantics. Next, we associate another semantics with $\varphi(\overline{\mathbf{u}}, \overline{\mathbf{y}})$, which captures a set of input-output signals corresponding to the input-output sequences captured by the predicate in the standard semantics. We will use bold letters such as $\overline{\mathbf{u}}$ and $\overline{\mathbf{y}}$ to denote variable names, and regular letters such as $\bar{u}$ and $\bar{y}$ to denote valuations for the variables.

Definition 7. Given $\theta \geq 0, r>0, \epsilon>0$, and predicate $\varphi(\overline{\boldsymbol{u}}, \overline{\boldsymbol{y}})$, $\llbracket \varphi(\overline{\boldsymbol{u}}, \bar{y}) \rrbracket^{(\theta, \epsilon, r)}$ be the set of all input-output signals $(u, y)$ such that $\|\dot{u}\| \leq \theta$ and there exists $(\bar{u}, \bar{y}) \in \llbracket \varphi(\bar{u}, \bar{y}) \rrbracket_{p w}$ which satisfy the following conditions: $[u]_{r}=\bar{u},\left\|y-\langle\bar{y}\rangle_{r}\right\| \leq \epsilon$. In other words, $\llbracket \varphi(\overline{\boldsymbol{u}}, \overline{\boldsymbol{y}}) \rrbracket^{(\theta, \epsilon, r)}=\left\{(u, y) \mid \exists(\bar{u}, \bar{y}) \in \llbracket \varphi(\overline{\boldsymbol{u}}, \overline{\boldsymbol{y}}) \rrbracket_{p w},\|\dot{u}\| \leq \theta,[u]_{r}=\right.$ $\left.\bar{u},\left\|y-\langle\bar{y}\rangle_{r}\right\| \leq \epsilon\right\}$.

Next, we define an abstraction predicate that captures all inputoutput sample points of the input-output trajectories corresponding to a system $\mathcal{S}$ with sample time $r$.

Definition 8. Given a system $\mathcal{S}=(I, O, \mathcal{U}, \mathcal{Y}, \mathcal{T})$ and $r>0$, a predicate $\phi_{\mathcal{S}}^{r}(\overline{\boldsymbol{u}}, \overline{\boldsymbol{y}})$ is an abstraction predicate if it captures all sequences $(\bar{u}, \bar{y})$ such that there exists an output signal $y$ satisfying $\left(\langle\bar{u}\rangle_{r}, y\right) \in \mathcal{T}$ and $[y]_{r}=\bar{y}$. In other words,

$$
\llbracket \phi_{\mathcal{S}}^{r} \rrbracket_{p w}=\left\{(\bar{u}, \bar{y}) \mid \exists y \text { such that }\left(\langle\bar{u}\rangle_{r}, y\right) \in \mathcal{T},[y]_{r}=\bar{y}\right\} .
$$

Note that Definition 8 does not provide a specific predicate, but specifies the set defined by the predicate in the standard semantics.
Our main objective is to find $\epsilon$ and $r$ such that $\llbracket \phi_{\mathcal{S}}^{r} \rrbracket^{(\theta, \epsilon, r)}$ is a $\gamma$ bounded over-approximation of $\mathcal{S}$ with respect to input signals whose rate of change is bounded by $\theta$. We define certain auxiliary functions for $\mathcal{S}$, which will help in establishing the values of $\epsilon$ and $r$, given $\theta$ and $\gamma$. First, we define an input-output value function that captures the maximum distance between two values of an output trajectory. These values are points of the output trajectory at two time points within a sample interval corresponds to a constant input signal.

Definition 9. Given a system $\mathcal{S}$, we call $C_{\mathcal{S}}: \mathbb{R}_{\geq 0} \rightarrow \mathbb{R}_{\geq 0}$, a constant input-output value function iffor any $r \geq 0$, for all $t \geq 0$, and for all $t_{1}, t_{2} \in[t, t+T]$ with $\left\|t_{1}-t_{2}\right\| \leq r$, for all $u \in \operatorname{Traj}(\mathcal{U})$ such that $\forall t \in\left[t_{1}, t_{2}\right], u(t)=u\left(t_{1}\right)$, and for all $y$ such that $(u, y) \in \mathcal{T}$, we have $\left\|y\left(t_{1}\right)-y\left(t_{2}\right)\right\| \leq C_{\mathcal{S}}(r)$.

Next, we want to capture the output behaviors when input signals are not constant. We define bounded input-output value function that expresses the maximum variation between two output trajectories when their corresponding input signals are within a certain value and the rate of one of the input signals is bounded by some assigned value.

Definition 10. Given a system $\mathcal{S}$, we name $\mathcal{B}_{\mathcal{S}}: \mathbb{R}_{\geq 0} \times \mathbb{R}_{\geq 0} \rightarrow$ $\mathbb{R}_{\geq 0}$, a bounded input-output value function if for any $\theta \geq 0, \delta \geq 0$, for any two trajectories $(u, y),\left(u^{\prime}, y^{\prime}\right) \in \mathcal{T}$ such that $\|\dot{u}\| \leq \theta$ and $\left\|u-u^{\prime}\right\| \leq \delta$, we have $\left\|y-y^{\prime}\right\| \leq \mathcal{B}_{\mathcal{S}}(\theta, \delta)$, where $y(0)=y^{\prime}(0)$.

Next, we state our main result, that is, given an error bound $\gamma$, we can compute an $\epsilon$ and an $r$, such that $\llbracket \phi_{\mathcal{S}}^{r} \rrbracket^{(\theta, \epsilon, r)}$ is a $\gamma$ bounded over-approximation of $\mathcal{S}$.

Theorem 1. Given system $\mathcal{S}, \gamma>0$ and $\theta>0$, constant inputoutput value function $C_{\mathcal{S}}$, bounded input-output value function $\mathcal{B}_{\mathcal{S}}$, let $r>0, \epsilon>0$ be such that $\epsilon=C_{\mathcal{S}}(r)+\mathcal{B}_{\mathcal{S}}(\theta, \theta r)$ and $2 \epsilon \leq \gamma$. Then we have $\llbracket \mathcal{S} \rrbracket^{\theta} \subseteq \llbracket \phi_{\mathcal{S}}^{r} \rrbracket^{(\theta, \epsilon, r)} \subseteq \llbracket \mathcal{S} \rrbracket^{(\theta, \gamma)}$.

Next, we define $\gamma$-bounded over-approximation for the composition of input-output systems.

\subsection{Compositional Abstraction}

The broad goal of this section is to define the abstraction predicate for a composed system in terms of its components. Recall that we constructed the abstraction predicate and the corresponding parameters $\epsilon$ and $r$ in the semantics depending on the error bound $\gamma$ and rate of change of input $\theta$. Note that when given a composed system $\mathcal{S}_{1} \triangleright \mathcal{S}_{2}$ and a bound on the rate of change of input $\theta$, the rate of change of input to $\mathcal{S}_{1}$ is still bounded by $\theta$. However, the input to $\mathcal{S}_{2}$ might consist of some outputs of $\mathcal{S}_{1}$ which do not obey the bound $\theta$. Hence, we need to compute an abstraction predicate of $\mathcal{S}_{2}$ with respect to a different bound on the rate of change of input. The bound is computed using a function called the output rate value function that expresses an upper bound on the rate of change of output for a given bound on the rate of change of input.

Definition 11. Given a system $\mathcal{S}$, and a value $\theta \geq 0$, we call $\mathcal{D}_{\mathcal{S}}: \mathbb{R}_{\geq 0} \rightarrow \mathbb{R}_{\geq 0}$, an output rate value function, if for any trajectory $(u, y) \in \mathcal{T}$ such that $\|\dot{u}\| \leq \theta$, we have $\|\dot{y}\| \leq \mathcal{D}_{\mathcal{S}}(\theta)$.

The next proposition capture the relation between input-output trajectories of the composed system and that of its components using the notion of output rate value function. 
Proposition 1. Given systems $\mathcal{S}_{1}$ and $\mathcal{S}_{2}$, rate $\theta \geq 0$, and output rate value function $\mathcal{D}_{\mathcal{S}_{1}}$, let $\mathcal{S}=\mathcal{S}_{1} \triangleright \mathcal{S}_{2}$. Then $\llbracket \mathcal{S} \rrbracket^{\theta}$ is the set of composition of trajectories $(u, w) \in \mathcal{T}_{1}$ and $(w, y) \in \mathcal{T}_{2}$ such that $(u, w) \in \llbracket \mathcal{S}_{1} \rrbracket^{\theta}$ and $(w, y) \in \llbracket \mathcal{S}_{2} \rrbracket^{\mathcal{D}_{\mathcal{S}_{1}}(\theta)}$. Alternatively,

$$
\llbracket \mathcal{S} \rrbracket^{\theta}=\left\{(u, y) \mid \exists w,(u, w) \in \llbracket \mathcal{S}_{1} \rrbracket^{\theta},(w, y) \in \llbracket \mathcal{S}_{2} \rrbracket^{\mathcal{D}_{\mathcal{S}_{1}}(\theta)}\right\} .
$$

Next, we define the main component of our compositional abstraction framework, namely, the construction of the abstraction of the composed system from that of the components.

Definition 12. Given $\epsilon \geq 0$, predicates $\varphi_{1}$ and $\varphi_{2}$, we define $\varphi_{1} \circ_{\epsilon} \varphi_{2}$ to be the predicate $\varphi_{1} \circ_{\epsilon} \varphi_{2}(\overline{\boldsymbol{u}}, \overline{\boldsymbol{y}})$ which is given by:

$$
\exists \overline{\boldsymbol{w}}, \overline{\boldsymbol{w}}^{\prime}, \varphi_{1}(\overline{\boldsymbol{u}}, \overline{\boldsymbol{w}}) \wedge \varphi_{2}\left(\overline{\boldsymbol{w}}^{\prime}, \overline{\boldsymbol{y}}\right) \wedge\left\|\overline{\boldsymbol{w}}-\overline{\boldsymbol{w}}^{\prime}\right\| \leq \epsilon .
$$

Next, we show that if we have abstraction predicates $\varphi_{1}$ and $\varphi_{2}$ for systems $\mathcal{S}_{1}$ and $\mathcal{S}_{2}$, respectively, which satisfy Theorem 1, then we can construct an abstraction predicate $\varphi$ as in Definition 12 that captures the behaviors of the composed system.

Theorem 2. Consider systems $\mathcal{S}_{1}$ and $\mathcal{S}_{2}$, predicates $\varphi_{1}$ and $\varphi_{2}$, and values $\theta, \epsilon_{1}, \epsilon_{2}, \gamma_{1}$ and $\gamma_{2}$ such that $\llbracket \mathcal{S}_{1} \rrbracket^{\theta} \subseteq \llbracket \varphi_{1} \rrbracket^{\left(\theta, \epsilon_{1}, r\right)} \subseteq$ $\llbracket \mathcal{S}_{1} \rrbracket^{\left(\theta, \gamma_{1}\right)}$ and $\llbracket \mathcal{S}_{2} \rrbracket^{\mathcal{D}_{\mathcal{S}_{1}}(\theta)} \subseteq \llbracket \varphi_{2} \rrbracket^{\left(\mathcal{D}_{\mathcal{S}_{1}}(\theta), \epsilon_{2}, r\right)} \subseteq \llbracket \mathcal{S}_{2} \rrbracket^{\left(\mathcal{D}_{\mathcal{S}_{1}}(\theta), \gamma_{2}\right)}$. Then, we have

$$
\llbracket \mathcal{S}_{1} \triangleright \mathcal{S}_{2} \rrbracket^{\theta} \subseteq \llbracket \varphi \rrbracket^{\left(\theta, \epsilon_{2}, r\right)} \subseteq \llbracket \mathcal{S}_{1} \triangleright \mathcal{S}_{2} \rrbracket^{(\theta, \gamma)} \text {, where }
$$

(a) $\varphi=\varphi_{1}{ }^{\circ} \epsilon_{1} \varphi_{2}$;

(b) $\gamma \geq 3 \gamma_{2}+\mathcal{B}_{\mathcal{S}_{2}}\left(\mathcal{D}_{\mathcal{S}_{1}}(\theta), \gamma_{1}+\mathcal{D}_{\mathcal{S}_{1}}(\theta) r\right)+B_{\mathcal{S}_{2}}\left(\mathcal{D}_{\mathcal{S}_{1}}(\theta), \gamma_{1}\right)$.

Finally, given an unsafe region $U_{R}$, we construct a predicate $\Psi_{\text {unsafe }}$ to express that the system is approximately unsafe as follows: $\exists(\bar{u}, \bar{w}) \in \llbracket \phi_{\mathcal{S}_{1}}^{r_{1}} \circ_{\epsilon_{1}} \phi_{\mathcal{S}_{2}}^{r_{2}} \rrbracket_{p w}, s \in U_{R}$, such that $\bigvee_{i=1}^{n} \| s-$ $\bar{w}_{i} \| \leq \epsilon_{2}$. Unsatisfiability of the predicate $\Psi_{\text {unsafe }}$ guarantees the safety of the composed system.

\section{CONCLUSIONS}

In this paper, we presented a compositional framework to compute bounded error approximations of composed systems, and instantiated that framework for the case of linear dynamical systems. Constructing compositional abstractions has computational overheads, however, compositionality provides additional benefits towards construction of abstraction as well as analysis. Firstly, if a particular component changes, we need to only recompute the abstraction for the component. Also, by treating the input-output abstractions as assumptions/guarantees for the components, we might be able to do away with re-analysis by just checking if the new component satisfies these assumptions and guarantees. In the future, we intend to extend the framework for non-linear and hybrid systems.

\section{ACKNOWLEDGEMENTS}

Pavithra Prabhakar was partially supported by NSF CAREER Award No. 1552668 and ONR YIP Award No. N000141712577.

\section{REFERENCES}

[1] R. Alur, C. Courcoubetis, N. Halbwachs, T.A. Henzinger, P.-H. Ho, X. Nicollin, A. Olivero, J. Sifakis, and S. Yovine. 1995. The algorithmic analysis of hybrid systems. Theoretical Computer Science.

[2] R. Alur, T. Dang, and F. Ivancic. 2003. Counter-Example Guided Predicate Abstraction of Hybrid Systems. In International Conference on Tools and Algorithms for the Construction and Analysis of Systems.
[3] Rajeev Alur, Thao Dang, and Franjo Ivancic. 2006. Predicate abstraction for reachability analysis of hybrid systems. ACM Transactions on Embedded Computing Systems.

[4] E. Asarin, T. Dang, and A. Girard. 2007. Hybridization methods for the analysis of nonlinear systems. Acta Informatica.

[5] Mihaela Gheorghiu Bobaru, Corina S. Pasareanu, and Dimitra Giannakopoulou. 2008. Automated Assume-Guarantee Reasoning by Abstraction Refinement. In International Conference on Computer Aided Verification.

[6] Sergiy Bogomolov, Goran Frehse, Marius Greitschus, Radu Grosu, Corina S. Pasareanu, Andreas Podelski, and Thomas Strump. 2014. Assume-Guarantee Abstraction Refinement Meets Hybrid Systems. In Haifa Verification Conference.

[7] Xin Chen, Erika Abraham, and Sriram Sankaranarayanan. 2012. Taylor Model Flowpipe Construction for Non-linear Hybrid Systems. In IEEE Real-Time Systems Symposium (RTSS).

[8] Yu-Fang Chen, Edmund M. Clarke, Azadeh Farzan, Ming-Hsien Tsai, Yih-Kuen Tsay, and Bow-Yaw Wang. 2010. Automated Assume-Guarantee Reasoning through Implicit Learning. In International Conference on Computer Aided Verification.

[9] Chris Chilton, Bengt Jonsson, and Marta Z. Kwiatkowska. 2014. Compositional assume-guarantee reasoning for input/output component theories. Sci. Comput. Program.

[10] A. Chutinan and B.H. Krogh. 2003. Computational techniques for hybrid system verification. IEEE Trans. Automat. Control.

[11] E.M. Clarke, A. Fehnker, Z. Han, B. Krogh, J. Ouaknine, O. Stursberg, and M. Theobald. 2003. Abstraction and Counterexample-Guided Refinement in Model Checking of Hybrid Systems. Journal on Foundations of Computer Science.

[12] T. Dang, O. Maler, and R. Testylier. 2010. Accurate hybridization of nonlinear systems. In International Conference on Hybrid Systems: Computation and Control.

[13] Goran Frehse, Colas Le Guernic, Alexandre Donzé, Scott Cotton, Rajarshi Ray, Olivier Lebeltel, Rodolfo Ripado, Antoine Girard, Thao Dang, and Oded Maler. 2011. SpaceEx: Scalable Verification of Hybrid Systems. In International Conference on Computer Aided Verification.

[14] A. Girard. 2005. Reachability of uncertain linear systems using zonotopes. In International Conference on Hybrid Systems: Computation and Control.

[15] Antoine Girard. 2013. A composition theorem for bisimulation functions. CoRR.

[16] Antoine Girard and George J. Pappas. 2007. Approximation Metrics for Discrete and Continuous Systems. IEEE Trans. Automat. Contr.

[17] T.A. Henzinger, P.W. Kopke, A. Puri, and P. Varaiya. 1995. What's decidable about hybrid automata?. In ACM Symposium on Theory of Computation.

[18] Thomas A. Henzinger. 1996. The Theory of Hybrid Automata. In IEEE Symposium on Logic in Computer Science.

[19] Ratan Lal and Pavithra Prabhakar. 2015. Bounded error flowpipe computation of parameterized linear systems. In International Conference on Embedded Software.

[20] Ratan Lal and Pavithra Prabhakar. 2017. Safety analysis using compositional bounded error approximations of communicating hybrid systems. In Annual Conference on Decision and Control.

[21] Alessio Lomuscio, Ben Strulo, Nigel G. Walker, and Peng Wu. 2013. AssumeGuarantee Reasoning with Local Specifications. Int. F. Found. Comput. Sci.

[22] Nancy A. Lynch, Roberto Segala, and Frits W. Vaandrager. 2003. Hybrid I/O automata. Inf. Comput.

[23] Wonhong Nam and Rajeev Alur. 2006. Learning-Based Symbolic AssumeGuarantee Reasoning with Automatic Decomposition. In International Symposium on Automated Technology for Verification and Analysis.

[24] André Platzer. 2011. Logic and Compositional Verification of Hybrid Systems (Invited Tutorial). In International Conference on Computer Aided Verification.

[25] Pavithra Prabhakar and Mahesh Viswanathan. 2011. A dynamic algorithm for approximate flow computations. In International Conference on Hybrid Systems: Computation and Control.

[26] Nima Roohi, Pavithra Prabhakar, and Mahesh Viswanathan. 2016. Hybridization Based CEGAR for Hybrid Automata with Affine Dynamics. In International Conference on Tools and Algorithms for the Construction and Analysis of Systems.

[27] Nima Roohi, Pavithra Prabhakar, and Mahesh Viswanathan. 2017. Robust Model Checking of Timed Automata under Clock Drifts. In International Conference on Tools and Algorithms for the Construction and Analysis of Systems.

[28] Matthias Rungger and Majid Zamani. 2015. Compositional construction of approximate abstractions. In International Conference on Hybrid Systems: Computation and Control.

[29] Paulo Tabuada, George J. Pappas, and Pedro U. Lima. 2004. Compositional Abstractions of Hybrid Control Systems. Discrete Event Dynamic Systems.

[30] Yuichi Tazaki and Jun-ichi Imura. 2008. Bisimilar Finite Abstractions of Interconnected Systems. In International Conference on Hybrid Systems: Computation and Control.

[31] Ashish Tiwari. 2008. Abstractions for hybrid systems. Formal Methods in System Design.

[32] L. van den Dries and C. Miller. 1994. On the real exponential field with restricted analytic functions. Israel fournal of MAthematics. 